\title{
On the Use of Auxiliary Receive Channels for Clutter Mitigation With Phased Array Weather Radars
}

\author{
Khoi D. Le, Robert D. Palmer, Senior Member, IEEE, Boon Leng Cheong, Tian-You Yu, \\ Guifu Zhang, Senior Member, IEEE, and Sebastian M. Torres, Member, IEEE
}

\begin{abstract}
Phased array radars (PARs) are attractive in weather surveillance primarily because of their capability to electronically steer. When combined with the recently developed beam multiplexing (BMX) technique, these radars can obtain very rapid update scans that are useful in monitoring severe weather. A consequence is that the small number of contiguous samples of the time series obtained can be a challenge for temporal/spectral filters used for clutter mitigation. As a result, the accurate extraction of weather signals can become the limiting performance barrier for PARs that employ BMX in clutter-dominated scattering fields. By exploiting the spatial correlation of the auxiliary channel signals, the effect of clutter contamination can be reduced in these conditions. In this paper, three spatial filtering techniques that used low-gain auxiliary receive channels are presented. The effect of clutter mitigation was studied using numerical simulations of a tornadic environment for changes in signal-to-noise ratio, clutter-to-signal ratio, number of time series samples, varying clutter spectral widths, and maximum weight constraints. Since such data are not currently available from a horizontally pointed phased array weather radar, experimental validation was applied to an existing data set from the turbulent eddy profiler, which is a vertically pointed PAR. Although preliminary, the results show promise for clutter mitigation with extremely short nonuniform sampling.
\end{abstract}

Index Terms-Adaptive arrays, adaptive signal processing, meteorological radar, phased array radar (PAR), radar clutter, radar interference, remote sensing.

\section{INTRODUCTION}

$\mathbf{T}$ HE NATIONAL Weather Radar Testbed (NWRT), which is located in Norman, Oklahoma, has a $10-\mathrm{cm}$ phased array radar (PAR) that became operational in 2003 [1]. The radar consists of an Aegis SPY-1A antenna and a Weather Sur-

Manuscript received August 14, 2007; revised February 6, 2008 and April 17, 2008. First published December 2, 2008; current version published December 17, 2008. This work was supported in part by the National Severe Storms Laboratory (NOAA/NSSL) under Cooperative Agreement NA17RJ1227.

K. D. Le is with the School of Electrical and Computer Engineering, The University of Oklahoma, Norman, OK 73072 USA, and also with the Atmospheric Radar Research Center, Norman, OK 73072-7307 USA (e-mail: khoi@ou.edu).

R. D. Palmer is with the School of Meteorology, The University of Oklahoma, Norman, OK 73072-73071 USA, and also with the Atmospheric Radar Research Center, Norman, OK 73072-7307 USA.

B. L. Cheong and G. Zhang are with the School of Meteorology, The University of Oklahoma, Norman, OK 73072-73071 USA.

T.-Y. Yu is with the School of Electrical and Computer Engineering, The University of Oklahoma, Norman, OK 73072 USA (e-mail: khoi@ ou.edu).

S. M. Torres is with the NOAA/OAR National Severe Storms Laboratory, Norman, OK 73072 USA, and also with the Cooperative Institute for Mesoscale Meteorological Studies, The University of Oklahoma, Norman, OK 73072 USA.

Color versions of one or more of the figures in this paper are available online at http://ieeexplore.ieee.org

Digital Object Identifier 10.1109/TGRS.2008.2001260 veillance Radar-88 Doppler (WSR-88D) transmitter. It serves to test the operational utility of PARs in monitoring the weather [1] and analyzing the evolution of severe storms to provide early warning. Examples of ongoing research at the testbed include beam multiplexing (BMX) [2], simultaneous aircraft tracking and weather surveillance [3], spaced-antenna interferometry to measure crossbeam wind and to detect/locate subvolume inhomogeneity [4], [5], monopulse processing [6], and the extraction of atmospheric refractivity from the scattering of ground targets [7]. Preliminary results of storm evolution [8] and microburst [9] comparisons between the PAR, WSR-88D, and Terminal Doppler Weather Radar are promising. The NWRT is quickly becoming an ideal resource for obtaining information on the effectiveness, advantage, and limitations of PARs under operational and research conditions for the surveillance of weather.

PARs, such as the NWRT PAR, are attractive in weather surveillance primarily because of their capability to electronically change their radiation pattern. Thus, they can steer the beam to any direction almost instantaneously. When combined with BMX, rapid update scanning strategies can be obtained without compromising data accuracy. According to Yu et al. [2], BMX can reduce the acquisition time up to four times when the received signal-to-noise ratio (SNR) is larger than $10 \mathrm{~dB}$. A consequence of BMX, however, is that the number of contiguous time samples is small due to the scanning strategy that involves resampling the scattering region over many revisits. The length of the time series obtained during each revisit becomes problematic when clutter and weather are present simultaneously because the number of samples is shorter than the impulse response of the clutter filter. The performance of conventional clutter filters in time/spectral domain is poor in this situation, and the filtered signal does not accurately represent that of the weather signal. Since PARs consist of multiple received elements, the spatial correlation between the elements can be exploited using spatial filtering to reduce the clutter contamination. A benefit of spatial filtering is that contiguous samples are not required. As a result, spatial filtering can be used in conjunction with BMX, or other short-dwell time techniques, to image the atmosphere and provide updates that accurately reflect the atmospheric field. In addition, spatial filtering can be used to mitigate nonstationary clutter from aircraft, birds, etc. [10].

The application of PARs for monitoring the atmosphere is not new. Vertically pointed UHF and VHF radars have been used to image features within the atmospheric boundary layer and the mesosphere-stratosphere-troposphere layers (see [11]-[13], 
and the references therein). Spatial processing techniques for atmospheric observations include the simple spatial Fourier transform [14] and more complex techniques such as the maximum entropy method [15] and the data-dependent algorithm of [16] and [17]. Some of the atmospheric features that were investigated include clear-air turbulence and precipitation [18], plasma irregularities in the equatorial $F$ region [15], structure of the polar mesosphere summer echoes [19], and airborne clutter in the boundary layer [10]. Moreover, phased arrays have been used since 1960 in other fields such as military radar, sonar, communication, geophysical exploration, astrophysical exploration, and biomedicine (see, for examples, [20]-[22], and the references therein). Although imaging using PARs is a mature concept, the application to weather surveillance is extremely limited with the only example being the passive mobile radar constructed by the Naval Post Graduate School [23].

The focus of this paper is the applicability of spatial filtering and phased array for weather observations when the number of contiguous samples is small. The content is particularly pertinent to BMX when the received output signal is contaminated by clutter. Although spatial filtering may be applied to all the receive elements of the phased array, the techniques that will be examined are limited to the partially adaptive array providing a computationally efficient alternative. This application is considered to be the next major hardware upgrade phase of the NWRT PAR. The outline of this paper is as follows. In Section II, an introduction to clutter mitigation using adaptive array processing is presented. Discussion of the benefits and drawbacks of the fully and partially adaptive arrays is made. Also presented are three adaptive algorithms and factors that affect the performance for the partially adaptive arrays. In Section III, the numerical setup and simulation results are presented. Factors such as SNR, clutter-to-signal ratio (CSR), number of points (NPTS), maximum weight constraint, and clutter spectral widths are examined. In Section IV, the three algorithms are validated using data from the turbulent eddy profiler (TEP) obtained from a PAR, and the results are presented. This paper is concluded in Section V.

\section{Clutter Mitigation Using AdAPTIVE ARRAy Processing}

In systems such as the WSR-88D, there is only one receiver and one channel output. Temporal filters [24], [25], spectral/ autocorrelation filters [26], [27], and fits of polarimetric characteristics [28] can be used to extract the weather signal when clutter is present. Other techniques that can be used include designing the antenna sitting positions [29] and statistical selection of processed data using clutter maps [30], [31]. However, these systems are limited when weather and clutter are not distinct in either the temporal, spectral, or polarimetric domain. A notorious example is clutter caused by wind turbines, which has proven to be a difficult challenge given its wide and largely unpredictable Doppler spread [32]. Fortunately, spatial filtering using phased arrays may hold some promise in the mitigation of this increasingly important problem.

Phased array systems are composed of multiple spaced antennas, and the correlation between the time series signals of

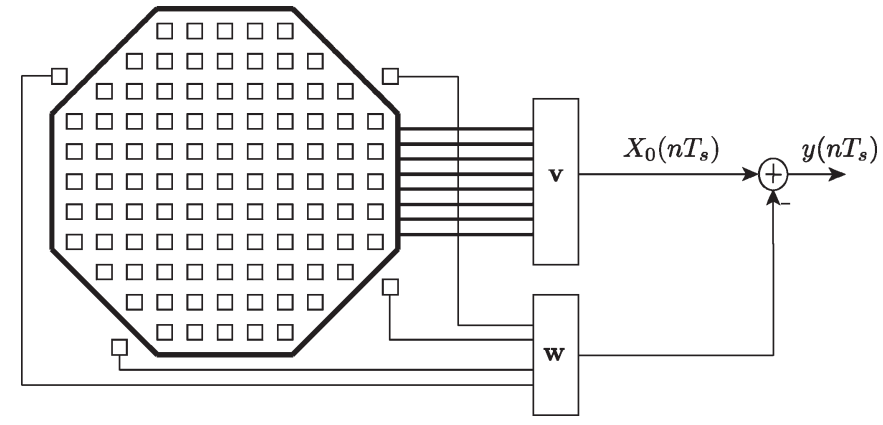

Fig. 1. Depiction of a partially adaptive array. The signal in the upper path is obtained by forming a nonadaptive radiation pattern in the steered direction with weights $\mathbf{v}$, and the lower channel is obtained using adaptive weights $\mathbf{w}$, which is selected by some optimization scheme. The output signal is obtained by subtracting the adaptive signal from the nonadaptive signal.

these sensors can be used to suppress the clutter when the weather and clutter are not separated in the temporal, spectral, or polarimetric domain. For example, the NWRT PAR consists of 4352 elements that make up the main receiver and six auxiliary elements used for canceling sidelobe clutter. These radars offer the capabilities to obtain radiation pattern on demand and degrade gracefully in performance. Processing methods for such systems are discussed in [33]-[37]. Additionally, they can adapt to the weather conditions, and changes can be made on a sample-by-sample or block-by-block basis. In the current WSR-88D, between 16 and 300 time samples are used to estimate the power and Doppler velocity. The number of contiguous samples collected decreases to as low as two samples when BMX is used.

Ideally, advanced systems such as fully adaptive arrays would be used for operational weather surveillance. However, systematic factors, such as designing the active/passive/hybrid structures, front-end architecture, array gain and noise figures, system dynamic range, array synthesis procedure, random and quantization errors, and heat management and power supplies, make the implementation difficult [38]. Partially adaptive arrays offer a simpler and lower cost alternate solution for monitoring the weather at the expense of mainlobe clutter mitigation. In the next section, a detailed description of these radars, their array models, and some of their spatially filtering techniques are presented.

\section{A. Partially Adaptive Arrays}

A partially adaptive array is an antenna system in which there are several auxiliary channel signals that can be individually weighted and a main channel signal. The main channel signal is obtained using a high-gain antenna, and the auxiliary channel signals are obtained using low-gain antennas. This array configuration was first introduced by Howells [39], and an example of such a configuration is shown in Fig. 1 for four auxiliary receive channels. The NWRT PAR is an example of such a system.

Accordingly, the output signal of the partially adaptive array is defined as

$$
y\left(n T_{\mathrm{s}}\right)=X_{0}\left(n T_{\mathrm{s}}\right)-\mathbf{w}^{\mathrm{H}} \mathbf{x}\left(n T_{\mathrm{s}}\right)
$$

where the output time series of the main channel is $X_{0}\left(n T_{\mathrm{s}}\right)$, the vector of time series of the auxiliary channel is $\mathbf{x}\left(n T_{\mathrm{s}}\right)$, and 
the weight vector of the auxiliary channel is $\mathbf{w}$. The time series and weight vectors are both column vectors, and the length of each vector is equal to the number of auxiliary channels. The Hermitian operator is $(\cdot)^{\mathrm{H}}$, and the sampling time is $T_{\mathrm{s}}$. Moreover, the output signal $\left\{\mathbf{x}\left(n T_{\mathrm{S}}\right) ; n=0,1,2, \ldots, N-1\right\}$ is assumed to be a sequence of random variables that is discrete in time. The goal of the algorithms discussed in the next sections will be to determine the optimal (in some sense) weight vector $\mathbf{w}_{\mathrm{O}}$.

In many adaptive schemes [36], [37], [40], [41], the output signal is also considered as the error signal, or $e\left(n T_{\mathrm{s}}\right)=$ $y\left(n T_{\mathrm{s}}\right)$, and its power is employed as a cost function. Additionally, the correlation matrix at zero temporal lag of the auxiliary channel, which is defined as

$$
\mathbf{R}=E\left\{\mathbf{x}\left(n T_{\mathrm{s}}\right) \mathbf{x}^{\mathrm{H}}\left(n T_{\mathrm{s}}\right)\right\}
$$

is used. The rank of $\mathbf{R}$ is an important parameter used in characterizing the inverse matrix that depends on the number of time series samples, the number of antenna elements, the noise level, and the number of strong scattering regions. Most often, diagonal loading [42] is applied to reduce the effects of distorted main beam shape and high sidelobes caused by inverting the smaller eigenvalues when calculating $\mathbf{R}^{-1}$. An indirect measure of diagonal loading is the maximum weight value $\epsilon=\max \|\mathbf{w}\|^{2}$.

1) Multiple sidelobe canceler (MSC): The MSC of [43] is a popular processing technique used in partially adaptive arrays that minimizes the expected mean-squared-error (mse) criteria to obtain the optimal filter weights. Thus, the minimization problem of the MSC is

$$
\min _{\mathbf{w}} E\left\{e\left(n T_{\mathrm{s}}\right) e^{*}\left(n T_{\mathrm{s}}\right)\right\} .
$$

In this system, the mse is positive definite, has a hyperparaboloid performance surface, and, therefore, has a global minimum solution. The solution could be obtained by taking derivatives or by applying the more elegant orthogonality principle, i.e., the solution is such that

$$
E\left\{\mathbf{x}\left(n T_{\mathrm{s}}\right) e^{*}\left(n T_{\mathrm{s}}\right)\right\}=\mathbf{0} .
$$

Substituting for the error and replacing $\mathbf{w}$ by the optimal weights $\mathbf{w}_{\mathbf{o}}$ gives

$$
E\left\{\mathbf{x}\left(n T_{\mathrm{s}}\right)\left(X_{0}^{*}\left(n T_{\mathrm{s}}\right)-\mathbf{x}^{\mathrm{H}}\left(n T_{\mathrm{s}}\right) \mathbf{w}_{\mathrm{o}}\right)\right\}=\mathbf{0} .
$$

Defining $\mathbf{p}=E\left\{\mathbf{x}\left(n T_{\mathrm{s}}\right) X_{0}^{*}\left(n T_{\mathrm{s}}\right)\right\}$ and rearranging provides the desired solution of

$$
\mathbf{w}_{\mathrm{o}}=\mathbf{R}^{-1} \mathbf{p}
$$

2) Minimum variance distortionless response (MVDR): The MVDR of [16] is a technique used to obtain the optimal filter weights that satisfy a unity gain constraint in the steered direction and produce an output signal with the minimum averaged power [41]. Its minimization criterion was modified in this work to apply the technique to the partially adaptive array configuration. The solution of this minimization can be obtained by solving either a quadratic minimization equation or by using the Lagrange multiplier method. Although the minimization problem of the MVDR is similar to that of the MSC, subtle differences exist, which will be outlined next.

The weights using MVDR are obtained by defining the averaged output power as

$$
E\left\{y\left(n T_{\mathrm{s}}\right) y^{*}\left(n T_{\mathrm{s}}\right)\right\}=E\left\{\left|X_{0}\left(n T_{\mathrm{s}}\right)-\mathbf{w}^{\mathrm{H}} \mathbf{x}\left(n T_{\mathrm{s}}\right)\right|^{2}\right\} .
$$

It follows that the minimization problem is

$$
\min _{\mathbf{w}} E\left\{\left|X_{0}\left(n T_{\mathrm{s}}\right)-\mathbf{w}^{\mathrm{H}} \mathbf{x}\left(n T_{\mathrm{s}}\right)\right|^{2}\right\} \text { subject to } \mathbf{w}^{\mathrm{H}} \mathbf{e}=0
$$

where the steering vector is e, which is simply the array response in that direction (note that there is no relationship between the steering vector and the error signal previously defined). Based on the constraint, it is desired that the output signal in the steered direction is left unaltered. Since $X_{0}(t)$ is not weighted, it is easier to solve the problem by defining

$$
\tilde{\mathbf{w}}=\left[\begin{array}{c}
w_{0} \\
-\mathbf{w}
\end{array}\right]
$$

and

$$
\tilde{\mathbf{R}}=E\left\{\left[\begin{array}{c}
X_{0}\left(n T_{\mathrm{s}}\right) \\
\mathbf{x}\left(n T_{\mathrm{s}}\right)
\end{array}\right]\left[\begin{array}{c}
X_{0}\left(n T_{\mathrm{s}}\right) \\
\mathbf{x}\left(n T_{\mathrm{s}}\right)
\end{array}\right]^{\mathrm{H}}\right\}
$$

The averaged output power is then

$$
\begin{aligned}
E\left\{y\left(n T_{\mathrm{s}}\right) y^{*}\left(n T_{\mathrm{s}}\right)\right\} & =E\left\{\left|\left[\begin{array}{c}
w_{0} \\
-\mathbf{w}
\end{array}\right]^{\mathrm{H}}\left[\begin{array}{c}
X_{0}\left(n T_{\mathrm{s}}\right) \\
\mathbf{x}\left(n T_{\mathrm{s}}\right)
\end{array}\right]\right|^{2}\right\} \\
& =\tilde{\mathbf{w}}^{\mathrm{H}} \tilde{\mathbf{R}} \tilde{\mathbf{w}} .
\end{aligned}
$$

Let

$$
\tilde{\mathbf{E}}=\left[\begin{array}{cc}
1 & 1 \\
-\mathbf{e} & \mathbf{0}
\end{array}\right]
$$

where $\mathbf{0}$ is a column zero vector with length equal to the number of auxiliary channels, and $\tilde{\mathbf{1}}=\left[\begin{array}{ll}1 & 1\end{array}\right]$. The constraint in the second column forces $w_{0}$ to be unity.

Using quadratic minimization to solve the problem

$$
\min _{\tilde{\mathbf{w}}} \tilde{\mathbf{w}}^{\mathrm{H}} \tilde{\mathbf{R}} \tilde{\mathbf{w}} \quad \text { subject to } \quad \tilde{\mathbf{w}}^{\mathrm{H}} \tilde{\mathbf{E}}=\tilde{\mathbf{1}}
$$

results in

$$
\tilde{\mathbf{w}}_{\mathrm{O}}=\tilde{\mathbf{R}}^{-1} \tilde{\mathbf{E}}\left(\tilde{\mathbf{E}}^{\mathrm{H}} \tilde{\mathbf{R}}^{-1} \tilde{\mathbf{E}}\right)^{-1} \tilde{\mathbf{1}}^{\mathrm{H}}
$$

3) Subspace tracking spatial projection (STSP): The STSP of [44], designed to mitigate radio frequency interference of phased array radio telescopes, is a technique used to obtain the optimal weights constrained to be in the subspace of the clutter and produces an output signal that is not composed of any clutter component. The optimal weights of the STSP are obtained by projecting the weights of the MSC onto the clutter subspace, defined by the correlation matrix $\mathbf{R}_{i}$.

The minimization problem is stated as follows:

$$
\min _{\mathbf{w}} E\left\{y\left(n T_{\mathrm{s}}\right) y^{*}\left(n T_{\mathrm{s}}\right)\right\} \quad \text { subject to } \quad \mathbf{w}_{\mathrm{o}} \in \mathbf{R}_{i} \text {. }
$$


In the minimization problem, $\mathbf{w}_{\mathrm{o}} \in \mathbf{R}_{i}$ implies that the output weights are a subspace of the clutter space. In [44], STSP was formulated to reduce the mainlobe distortion and high sidelobe levels produced by the optimal weights obtained from the MVDR technique. In this work, the methodology is applied to the optimal weights obtained from the MSC. Thus

$$
\mathbf{w}_{\mathrm{o}}=\mathbf{P}_{i}^{\|} \mathbf{R}^{-1} \mathbf{p}
$$

where $\mathbf{P}_{i}^{\|}=\mathbf{U}_{i} \mathbf{U}_{i}^{\dagger}$, with $\mathbf{U}_{i}^{\dagger}=\left(\mathbf{U}_{i}^{\mathrm{H}} \mathbf{U}_{i}\right)^{-1} \mathbf{U}_{i}^{\mathrm{H}}$ and $\mathbf{U}_{i}$ being the clutter eigenvectors.

At this point, three different techniques have been presented for obtaining the optimal weights of a partially adaptive array. In the next section, their performance of mitigating clutter and preserving weather is examined.

\section{Numerical Simulations}

The performance of the three sidelobe canceling (SLC) techniques is investigated via numerical simulations in terms of their capability to mitigate clutter and preserve weather. It will be evaluated for the parameter variations of $\epsilon, \mathrm{CSR}, \mathrm{SNR}$, NPTS, and standard deviation of clutter spectral widths $\sigma_{\mathrm{c}}$. In these simulations, CSR and SNR are defined as the ratio of the two powers measured at $0.5^{\circ}$ by the main receiver. NPTS is defined as the number of points used in each block to update the SLC weights. The retrieved power and Doppler velocity at each position are obtained using 256 points. The measures used are the bias and variance of the retrieved power as compared with the uncontaminated power. In addition, the performance is investigated for elevation angles ranging from $0.5^{\circ}$ to $4.5^{\circ}$ at $0.5^{\circ}$ interval.

\section{A. Radar Configuration and Environmental Fields}

The simulator of [45] was used in generating the time series signals. An illustration depicting the simulator is shown in Fig. 2. This simulator uses point targets to model the environmental and clutter fields. The point targets that emulate the weather field have scattering and dynamic properties obtained from the Advanced Regional Prediction System (ARPS) [46], [47]. In contrast, the reflectivity is constant, and the motion is random (as measured using $\sigma_{\mathrm{c}}$ ) for the point targets that emulate the ground clutter field. Control of the CSR is obtained by simulating the weather signal, setting the average weather signal power at $0.5^{\circ}$ to an arbitrary level, and then varying the power of the clutter signal at $0.5^{\circ}$ to obtain the desired CSR.

The weather field simulated is a tornadic event initiated using the 20 May 1977 Del City, Oklahoma, upper air sounding [48]. Parameters used in the simulation are listed in Table I. Fifty thousand point targets were used to model the environmental field, and 1000 point targets were used to model the clutter field. These targets occupied a volume of approximately $7 \times 7 \times$ $5 \mathrm{~km}^{3}$ with the radar located approximately $33 \mathrm{~km}$ from this volume. The positions of the elements of the radar are shown in

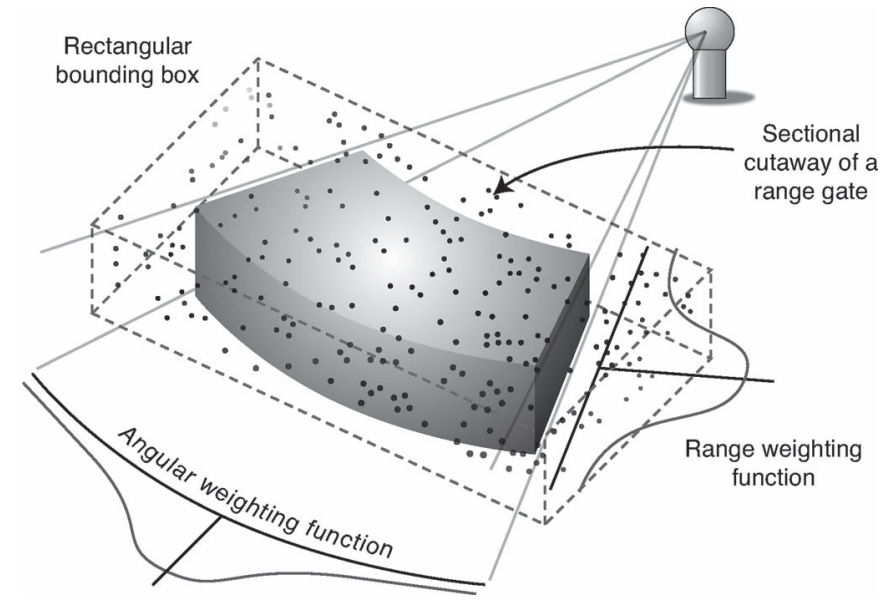

Fig. 2. Illustrated depiction of simulator [45]. Point source scatterers are used to emulate the scattering field. The point targets that emulate the weather field have scattering and dynamic properties obtained from the ARPS model. In contrast, the reflectivity is constant, and the motion is random for the point targets that emulate the ground clutter field.

TABLE I

SIMULATION PARAMETERS

\begin{tabular}{|c|c|}
\hline Simulated volume (Zonal x Meridional x AGL) & $7 \times 7 \times 5 \mathrm{~km}^{3}$ \\
\hline Number of atmospheric scatterers & 50,000 \\
\hline characteristics & ARPS-derived \\
\hline $\begin{array}{c}\begin{array}{c}\text { Number of ground clutter scatterers } \\
\text { characteristics }\end{array}\end{array}$ & $\begin{array}{l}1,000 \\
\text { constant reflectivity, } \\
\text { random motion }\end{array}$ \\
\hline Transmit antenna beamwidth & $20^{\circ}$ \\
\hline Receive aperture (width $\mathrm{x}$ height) & $3.6 \times 3.8 \mathrm{~m}^{2}$ \\
\hline Number of main array elements & 4352 \\
\hline Number of auxiliary elements & 6 \\
\hline Pulse repetition frequency & $1000 \mathrm{~Hz}$ \\
\hline Frequency & $3 \mathrm{GHz}$ \\
\hline Aliasing velocity & $23.4 \mathrm{~m} \mathrm{~s}^{-1}$ \\
\hline Number of pulses & 256 \\
\hline Range resolution & $235 \mathrm{~m}$ \\
\hline
\end{tabular}

Fig. 3. The main receiver is composed of 4352 elements, and the sidelobe canceler is composed of six auxiliary elements situated around the main receiver. The aperture of the combined radar is approximately $3.6 \times 3.8 \mathrm{~m}^{2}$, and its receive beamwidth is approximately $1.6^{\circ}$ at broadside, whereas its transmit beamwidth was selected to be $20^{\circ}$. The range resolution was $235 \mathrm{~m}$ while the aliasing velocity was $23.4 \mathrm{~m} \cdot \mathrm{s}^{-1}$ using a PRF of $10^{3} \mathrm{~Hz}$ at $\lambda=0.1 \mathrm{~m}$. The radar observed the simulated tornadic event over a 200-s duration at 25-s interval; 256 time series samples were collected during each scan.

Fig. 4 contains the uncontaminated power and Doppler velocity fields as retrieved by the simulated radar over the observed duration. In particular, the Doppler velocity field is retrieved using the pulse pair algorithm. The observed weather feature is the mesocyclone as it develops a classic hook echo signature observed in tornadic supercells. Fig. 5 contains the same fields but with clutter contamination. The parameters of this simulation are CSR $=30 \mathrm{~dB}, \mathrm{SNR}=70 \mathrm{~dB}$, and $\sigma_{\mathrm{c}}=$ $0.1 \mathrm{~m} \cdot \mathrm{s}^{-1}$. In later simulations, the CSR ranged from 0 to $50 \mathrm{~dB}$ and is approximately near the suppression level of the ground clutter filter (GCF) used in the WSR-88D [29]. As observed, the clutter power and its Doppler velocity mask those of the weather when clutter exists. The retrieved power is larger, 


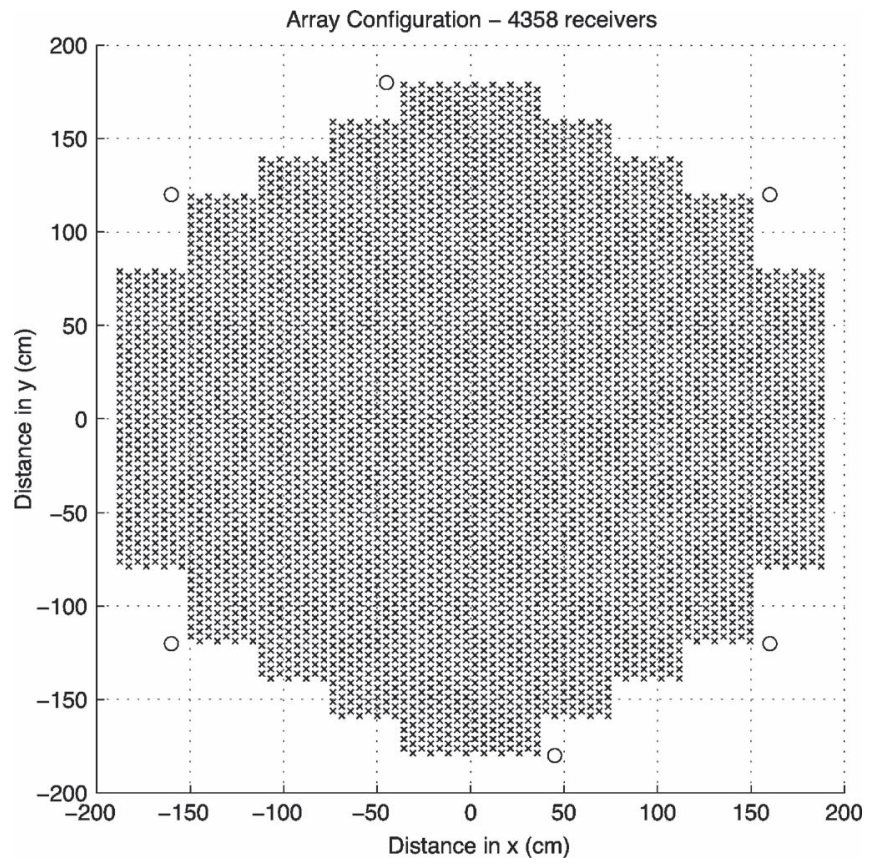

Fig. 3. Positions of receiving elements. The array consists of 4352 elements $(x)$ that make up the main channel and six auxiliary elements $(0)$. The aperture of the array is approximately $3.6 \mathrm{~m} \times 3.8 \mathrm{~m}$ (width $\times$ height), producing a beamwidth of $\approx 1.6^{\circ}$ at broadside.

and the measured Doppler velocity is close to zero. Only at $3.5^{\circ}$ are some features of the weather features evident.

\section{B. Statistical Comparison of Clutter Mitigation Schemes}

In the previous section, the uncontaminated retrieved power and Doppler velocity were presented along with the contaminated fields for $\mathrm{CSR}=30 \mathrm{~dB}, \mathrm{SNR}=70 \mathrm{~dB}$, and $\sigma_{\mathrm{c}}=0.1 \mathrm{~m} \cdot \mathrm{s}^{-1}$. The results show that the power and Doppler velocity fields of the weather were masked using only Fourier beamforming for this particular clutter case. In this section, the performance of the SLC techniques is investigated. By using this particular clutter scenario with $\epsilon=0.1$ and NPTS $=8$ as the standard case, the performance is examined for systematic changes in CSR, SNR, NPTS, $\epsilon$, and $\sigma_{\mathrm{c}}$.

The performance of the SLC techniques is determined by several controllable factors, and one such factor is the diagonal loading value. The results of the retrieved fields using MVDR at $t=100 \mathrm{~s}$ and $3.5^{\circ}$ for three amount of diagonal weights are shown in Fig. 6. The simulation parameters are $\mathrm{CSR}=30 \mathrm{~dB}$, $\mathrm{SNR}=70 \mathrm{~dB}, \sigma_{\mathrm{c}}=0.1 \mathrm{~m} \cdot \mathrm{s}^{-1}$, and NPTS $=4$. When the diagonal value is insufficient, both the clutter and weather signals are removed, and a negative bias in the power is observed. When the diagonal value is too large, there is insufficient clutter suppression, and residual clutter exists.

Another controllable factor is the clutter space used in STSP. Since this range is not known a priori, then it must be the eigenvector of the largest eigenvalue of $\mathbf{R}$ by assuming that the clutter is the dominant signal measured at the radar. Observe in Fig. 7 the retrieved fields obtained using STSP by removing the signal belonging to the subspace of the two largest eigenvalues. The simulation parameters are $\epsilon=0.1, \mathrm{CSR}=30 \mathrm{~dB}, \mathrm{SNR}=$ $70 \mathrm{~dB}, \sigma_{\mathrm{c}}=0.1 \mathrm{~m} \cdot \mathrm{s}^{-1}$, and NPTS $=4$. The retrieved results using the largest eigen subspace (eig-1) appear to be most like that of the weather, whereas the retrieved results using the second largest eigen subspace appear to be like that of the clutter. These results imply that most of the clutter signal belongs in the first eigen subspace and reinforce the assumption of the clutter being the dominant signal. However, complete removal of the clutter is only achieved if its subspace and that of the weather are orthogonal.

The results of the performance evaluation for the variations in $\epsilon$, CSR, SNR, NPTS, and $\sigma_{\mathrm{c}}$ are shown in Fig. 8. When $\epsilon$ was varied and decreased from 0.5 to 0.01 , as shown in Fig. 8(a), the performance at the higher elevation angles improved slightly for MVDR and MSC, whereas it remained approximately constant for STSP. The change observed using MVDR and MSC indicated a decrease in the loss of the weather signal with the increased $\epsilon$. In contrast, the performance degraded with decreased $\epsilon$ at the lower elevation angles for all the techniques and resulted in a larger residual clutter level. Since the elevation angle, the sidelobe level, and the clutter position are indirectly related, these results showed that a balance between $\epsilon$ and elevation angles was needed to retrieve the weather signal. The results are also shown in Fig. 8(b) for CSR $=0,10,30$, and $50 \mathrm{~dB}$. A decreasing trend was observed for MVDR and MSC as CSR was increased, and this indicated that the performance of these two techniques degraded for the same processing parameters. The performance improved for STSP when CSR increased from 0 to $10 \mathrm{~dB}$, decreased from 10 to $30 \mathrm{~dB}$, and significantly decreased from 30 to $50 \mathrm{~dB}$. Furthermore, a negative bias was observed for STSP when CSR $=0 \mathrm{~dB}$. These results implied that the dominant subspace at $\mathrm{CSR}=0 \mathrm{~dB}$ belonged to that of the weather and that the weather signal was being subtracted out. The dominant subspace transitioned from weather to clutter as CSR increased up to $30 \mathrm{~dB}$, and this resulted in better bias values. The range of the clutter subspace increased beyond the dominant subspace as the performance degraded when CSR increased from 30 to $50 \mathrm{~dB}$. The results of varying SNR between 10, 30, 50, and $70 \mathrm{~dB}$ in Fig. 8(c) showed that the performance remained constant at the higher SNR levels and was affected only when the SNR level fell to $10 \mathrm{~dB}$. The results were more variable with NPTS in Fig. 8(d). The performance of MVDR and MSC improved, whereas it degraded for STSP when NPTS increased from 8 to 32. Large negative biases and standard deviations were observed at NPTS $=4$ for MVDR and MSC, whereas the results remained approximately the same as compared for NPTS $=8$ and STSP. These results obtained coincided with the rank of $\mathbf{R}$ and $\tilde{\mathbf{R}}$. At low NPTS, these matrices are not full rank, and MVDR and MSC required large diagonal loading values to retrieve the weather signal. The matrices are full rank at large NPTS and required lower diagonal loading values. However, this condition affected the characterization of the clutter signal using STSP and degraded its performance. A similar subspace effect was observed in Fig. 8(e) when $\sigma_{\mathrm{c}}$ was varied from 0.01 to $0.5 \mathrm{~m} \cdot \mathrm{s}^{-1}$ as the physical relationship of $\sigma_{\mathrm{c}}$ is directly related to the localized position of the clutter source and the spread of the clutter subspace. As a result, the standard deviation and bias increased as $\sigma_{\mathrm{c}}$ increased with the performance being most significantly affected for STSP when $\sigma_{\mathrm{c}}$ changed from 0.1 to $0.5 \mathrm{~m} \cdot \mathrm{s}^{-1}$. 

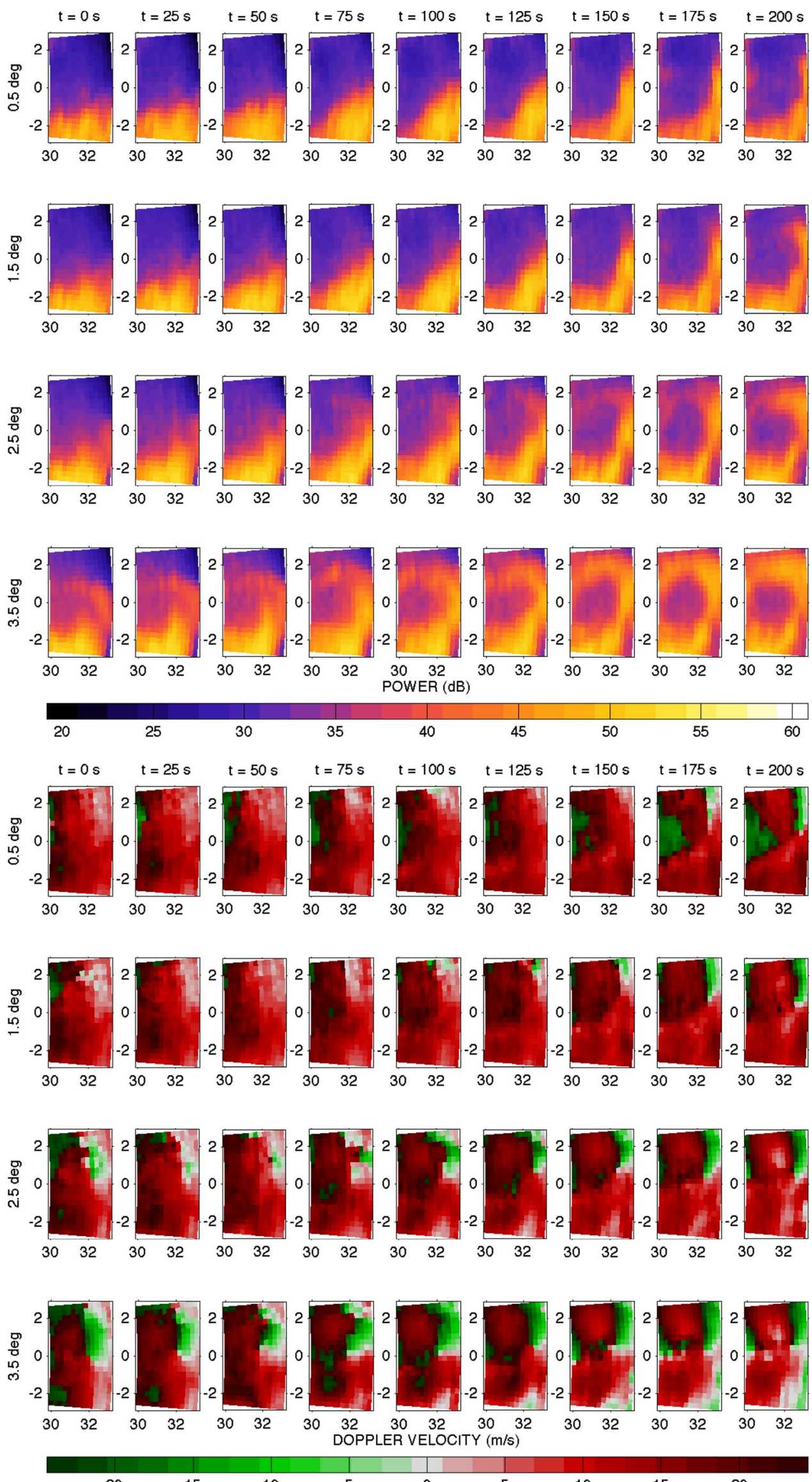

$-20$

$-15$

$-10$

$-5$

10

15

20

Fig. 4. Uncontaminated weather field. Observation fields retrieved using Fourier weighting on the main elements over a 200-s period. The observed feature is that of an evolving mesocyclone as it develops a strong signature of a hook echo. 

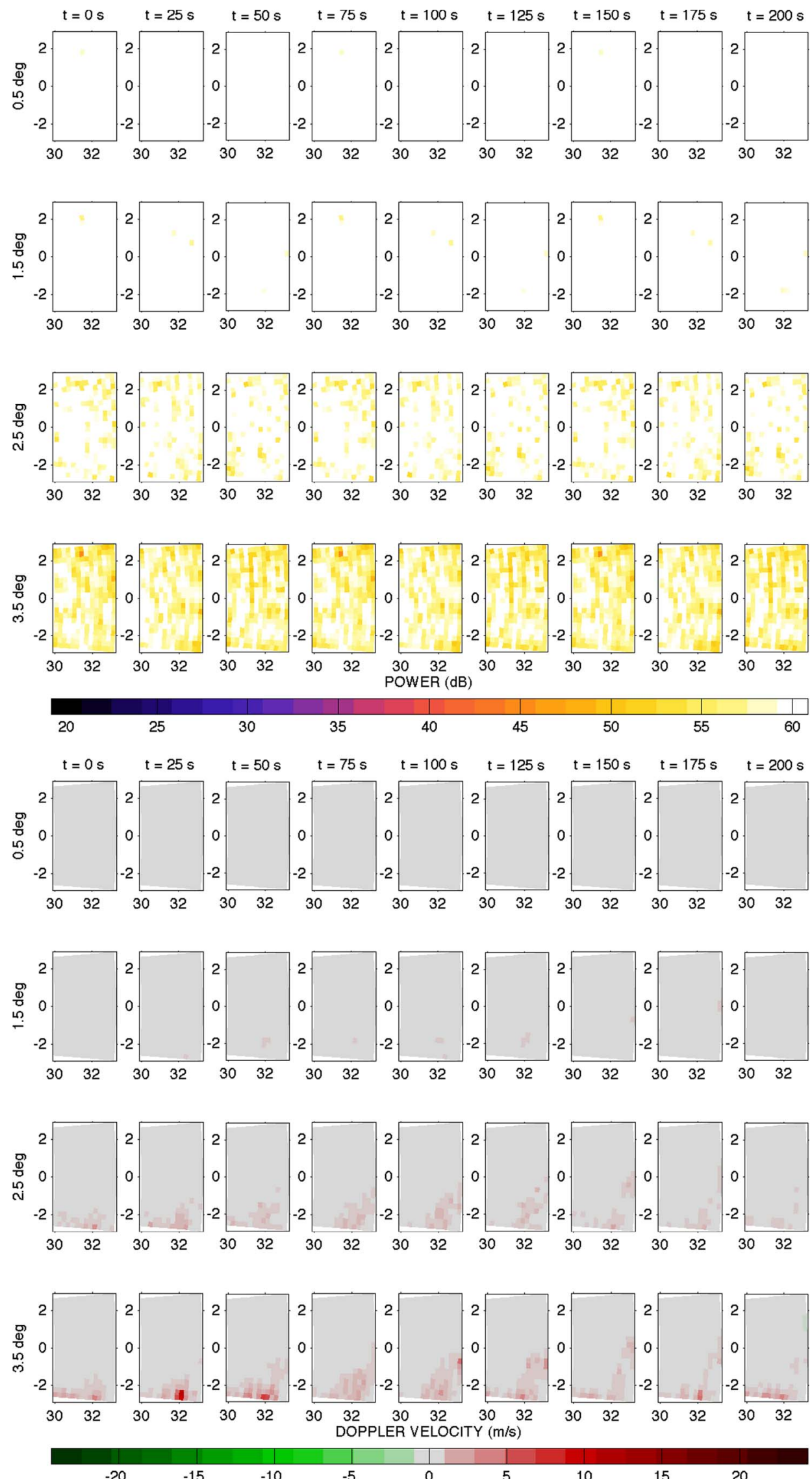

Fig. 5. Contaminated weather field. Retrieved field obtained using Fourier weighting on the main elements over a 200-s period. The simulation parameters are $\mathrm{CSR}=30 \mathrm{~dB}, \mathrm{SNR}=70 \mathrm{~dB}$, and $\sigma_{\mathrm{c}}=0.1 \mathrm{~m} \cdot \mathrm{s}^{-1}$. Observe that the environmental power field is masked by the strong return of the clutter and that the environmental Doppler velocity field is masked by the stationary characteristics of the clutter. 

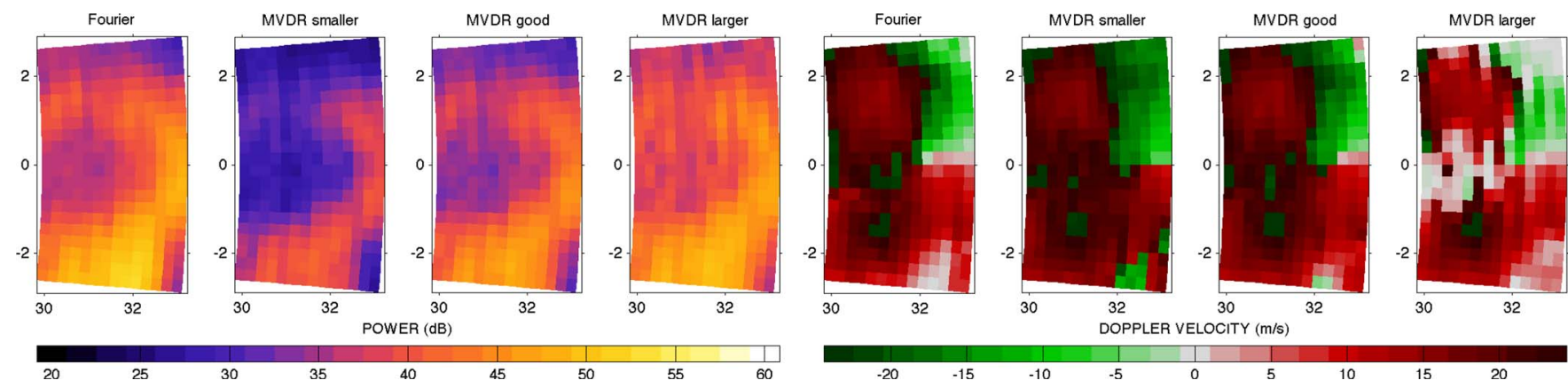

Fig. 6. Example of diagonal loading effect. Simulation parameters are $\mathrm{CSR}=30 \mathrm{~dB}, \mathrm{SNR}=70 \mathrm{~dB}, \sigma_{\mathrm{c}}=0.1 \mathrm{~m} \cdot \mathrm{s}^{-1}$, and NPTS $=4$. The fields were obtained at $t=100 \mathrm{~s}$ at $3.5^{\circ}$. The retrieved weather fields are like the uncontaminated fields obtained using the Fourier weights at the optimal diagonal loading value, whereas they contained a loss of the weather signal with a smaller value and residual clutter with a larger value.
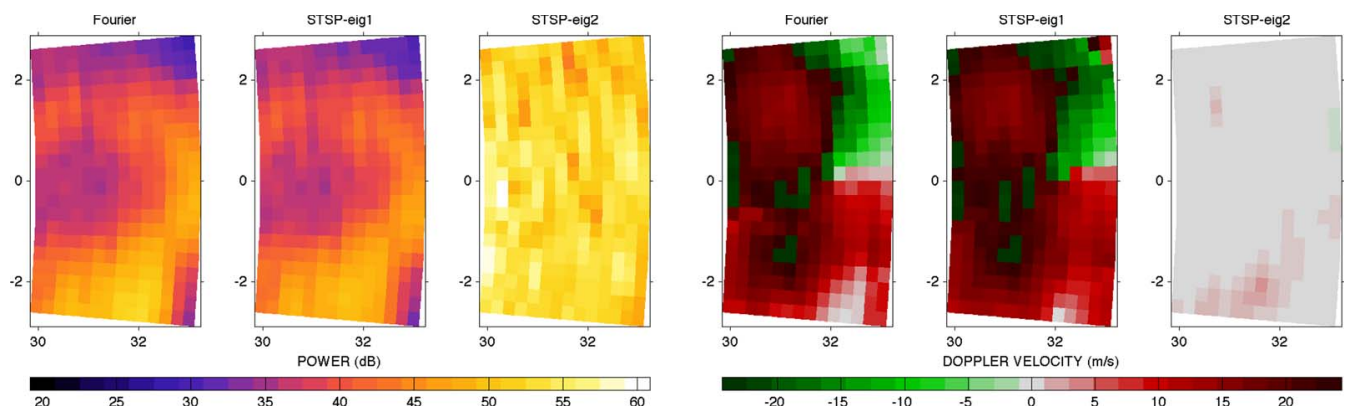

Fig. 7. Example of subspace selection effect. Simulation parameters are $\epsilon=0.1, \mathrm{CSR}=30 \mathrm{~dB}, \mathrm{SNR}=70 \mathrm{~dB}, \sigma_{\mathrm{c}}=0.1 \mathrm{~m} \cdot \mathrm{s}^{-1}$, and NPTS $=4$. The fields were obtained at $t=100 \mathrm{~s}$ at $3.5^{\circ}$. The fields obtained by subtracting the signal contained in the dominant subspace resemble the uncontaminated weather signal, whereas the fields obtained by subtracting the signal contained in the next dominant subspace resemble the clutter signal. These results imply that the dominant subspace is that of the clutter field.

\section{Algorithm Validation Using the TEP}

In this section, the conclusions drawn using simulations are validated using the TEP [49], a vertically pointed PAR, with data collected between 14:35 and 14:57 UTC on June 15, 2003 [10], [11]. The length of the data that are processed is approximately $20 \mathrm{~min}$, and the weather features that are observed are of boundary layer columnar updrafts and diffuse downdrafts. Power and Doppler velocity fields are presented for outputs obtained using the MVDR, the MSC, the STSP, and the simple spatial Fourier transform. Additionally, the fields are also presented for data obtained by processing the output signal of the receiver setup using the simple spatial Fourier transform with a regression clutter filter. The output signals obtained by applying the MVDR, the MSC, the STSP, and the simple spatial Fourier transform provide a qualitative measurement of the performance for a real-data atmospheric sensing case even though the TEP is not a horizontally pointing radar.

\section{A. Radar Configuration}

The TEP, as shown in Fig. 9, is a 915-MHz boundary layer imaging radar. This radar has been used in the Vertical Transport and Mixing campaign [50] as well as for the qualitative comparison of large eddy simulations to boundary layer observations [51]. The radar consists of a $25^{\circ}$ beamwidth transmit horn antenna and 64 receive $32^{\circ}$ beamwidth microstrip patch antennas.

Its pulse repetition frequency is $35 \mathrm{kHz}$; however, 250 coherent samples were integrated to produce an effective sampling time of $7.14 \mathrm{~ms}$ and an aliasing velocity of $11.48 \mathrm{~m} \cdot \mathrm{s}^{-1}$. The range resolution of this radar is approximately $33 \mathrm{~m}$ with 58 gates being recorded.

The array configuration of the radar in this particular experiment is shown in the right panel of Fig. 9. The elements are placed in a hexagonal configuration, and the aperture of the array is approximately $4.5 \times 4.0\left(\mathrm{~m}^{2}\right)$. The main channel consists of 52 elements marked by $\times$, and the sidelobe canceler consists of six elements marked by $\circ$. More details of the experimental configuration can be found in [10].

\section{B. Preliminary Results}

Time-height profile images of the power and Doppler velocity fields are shown in Fig. 10. These data were recorded approximately between 14:35 and 14:57 UTC on June 15, 2003, and processed using the MSC, the MVDR, the STSP, and the simple spatial Fourier transform. In addition, the images are presented by subtracting the mean of the time series samples (because of the small NPTS) to emulate a GCF. The controlled parameters used to process the data are NPTS $=4$ and $\epsilon=$ 0.01 . The small NPTS is used to emulate the condition of BMX. A total of 256 time samples were processed for each gate to produce the images. As in the simulations, the first eigenvector is used in the STSP technique as an approximation of the clutter subspace. The weather features observed in the images are clear air echoes of columnar updrafts, which are associated with superadiabitic heating at the surface, and diffuse downdrafts, which are associated with entrainment at the boundary layer. Nonweather features include echoes of aerial 
(a)

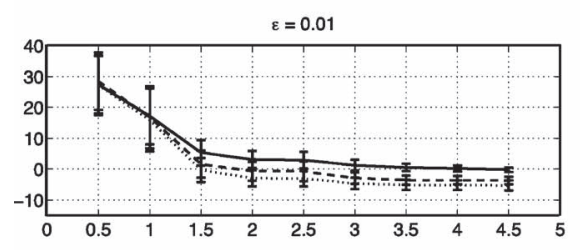

$\varepsilon=0.05$

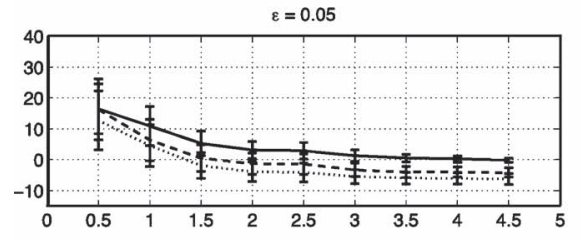

$\varepsilon=0.1$

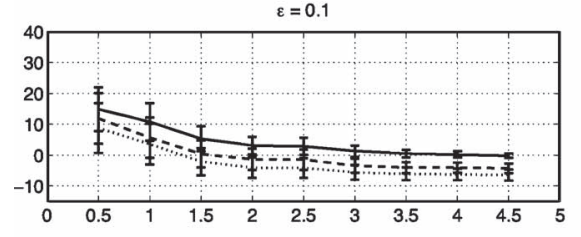

$\varepsilon=0.5$

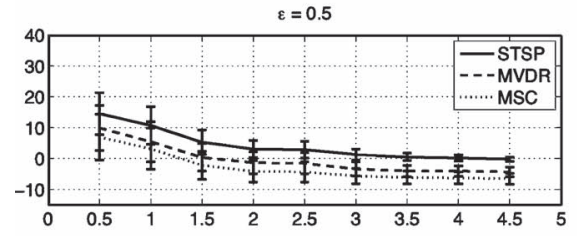

(d)

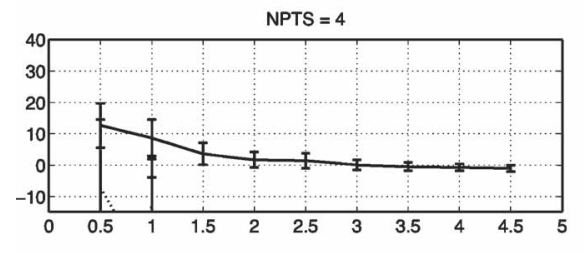

NPTS $=8$

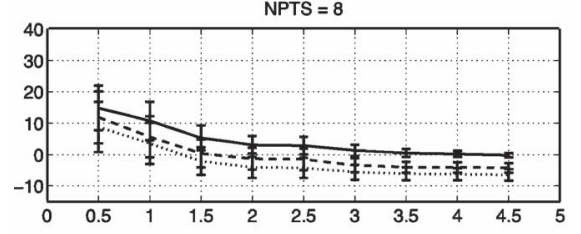

NPTS $=16$

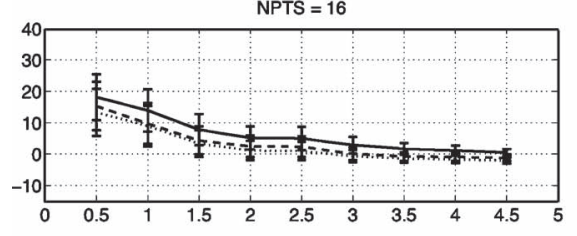

NPTS $=32$

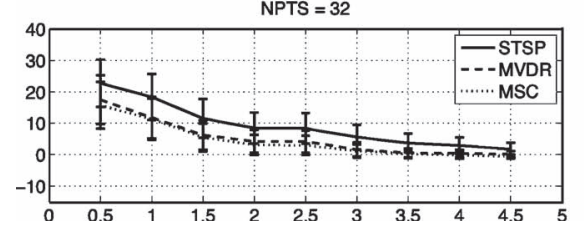

(b)

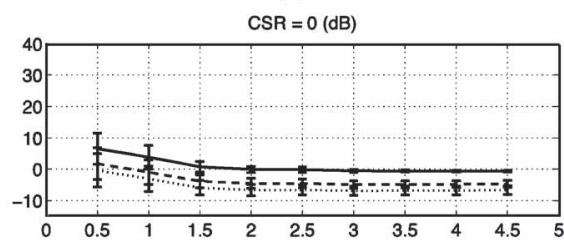

$\mathrm{CSR}=10(\mathrm{~dB})$

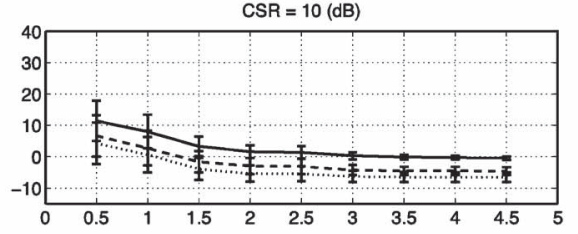

$\mathrm{CSR}=30(\mathrm{~dB})$

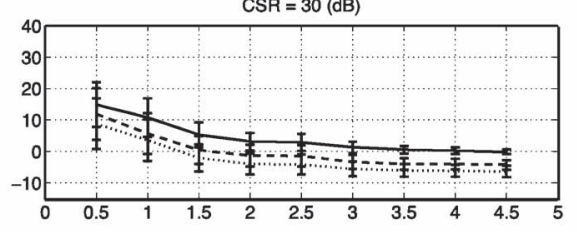

$\mathrm{CSR}=\mathbf{5 0}(\mathrm{dB})$

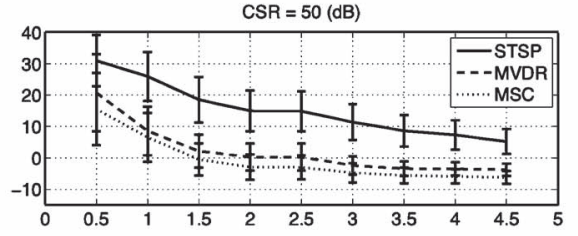

(e)

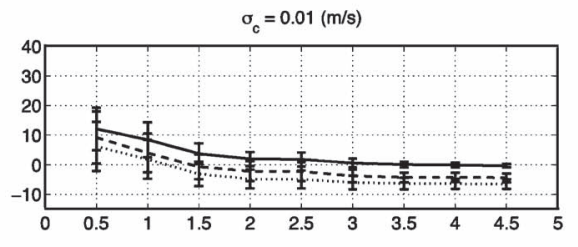

$\sigma_{c}=0.05(\mathrm{~m} / \mathrm{s})$

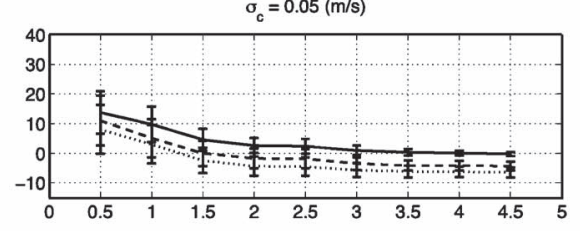

$\sigma_{c}=0.1(\mathrm{~m} / \mathrm{s})$

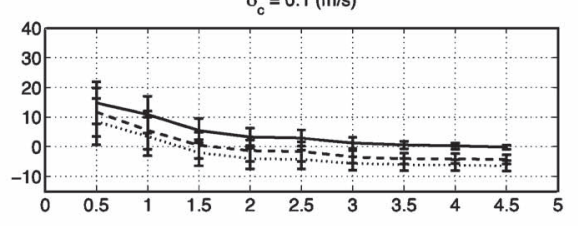

$\sigma_{c}=0.5(\mathrm{~m} / \mathrm{s})$

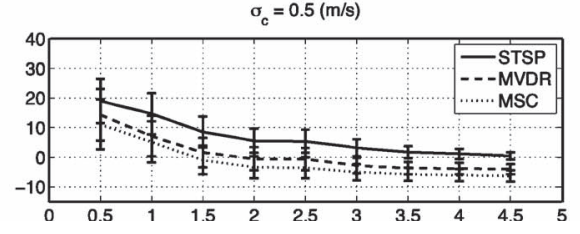

(c)
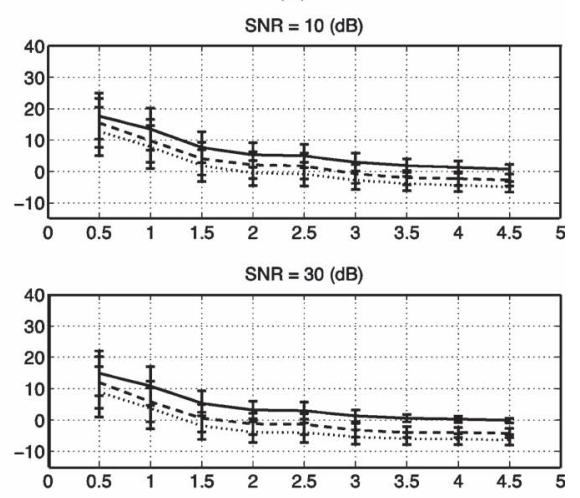

$\mathrm{SNR}=50(\mathrm{~dB})$

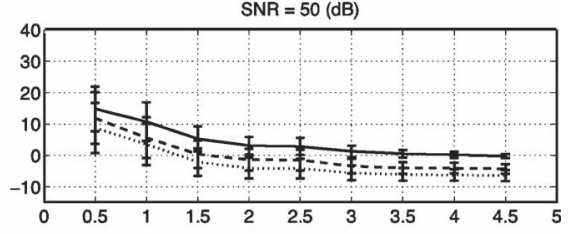

$\mathrm{SNR}=70(\mathrm{~dB})$

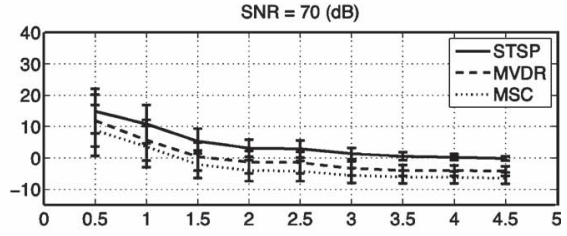

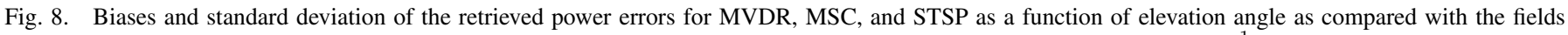
obtained without clutter. The standard conditions are $\mathrm{CSR}=30 \mathrm{~dB}, \mathrm{SNR}=70 \mathrm{~dB}, \mathrm{NPTS}=8, \epsilon=0.1$, and $\sigma_{\mathrm{C}}=0.1 \mathrm{~m} \cdot \mathrm{s}^{-1}$.

targets, ground targets, and intermittent interference. Examples of aerial targets are observed at approximately 14:50 UTC and $500 \mathrm{~m}$ and 14:49 UTC and $1250 \mathrm{~m}$, and they are characterized by parabolic temporal power and radial velocity couplet. Close inspection of the aerial target reveals that the upper target was attenuated almost completely by the three SLC techniques and that only the extrema of paraboloid of the lower target are strongly attenuated. Based on the attenuation characteristics, it is expected that the upper target is outside the mainlobe and that the lower target is inside the mainlobe. Examples 

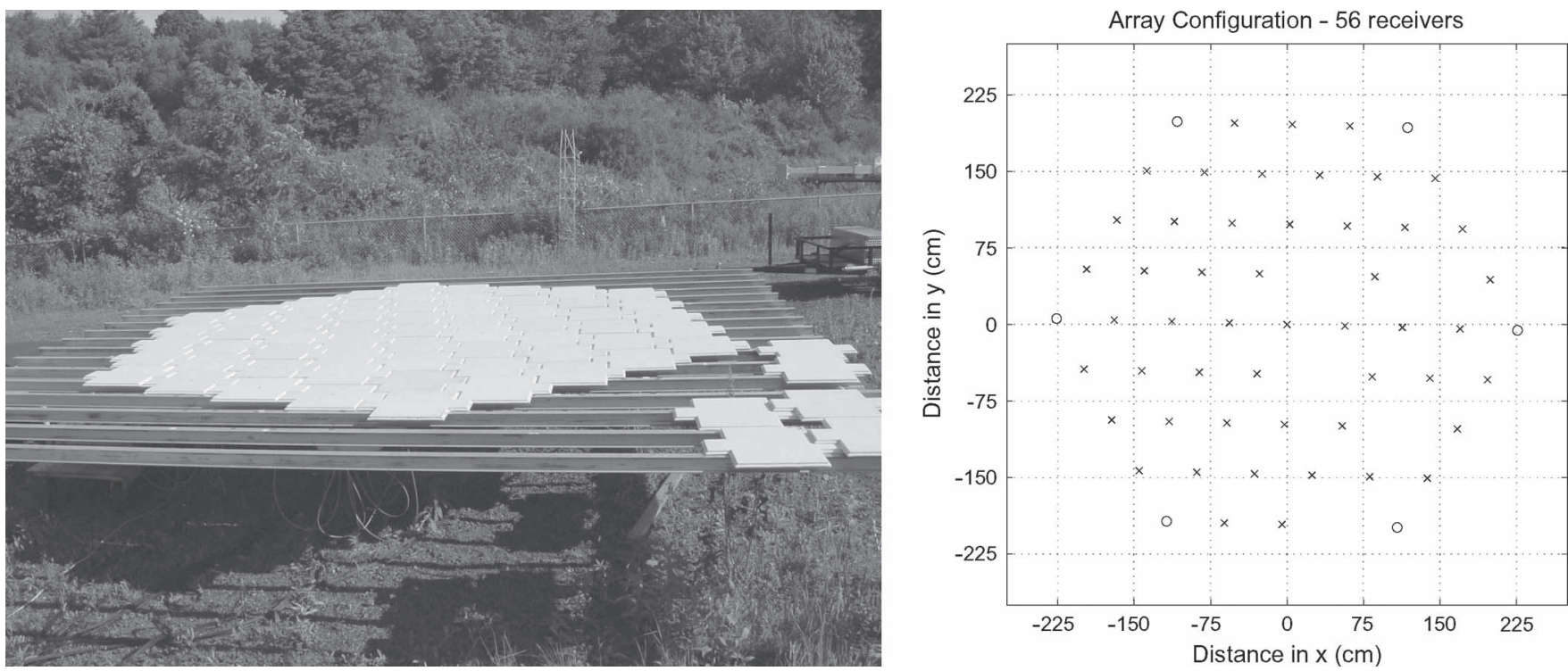

Fig. 9. TEP configuration. Left image is of the TEP. Right plot is of the TEP array configuration. The elements making up the main channel are marked $\times$, and the elements making up the auxiliary channels are marked $\circ$. Only the receivers that were working during the data collection are marked.
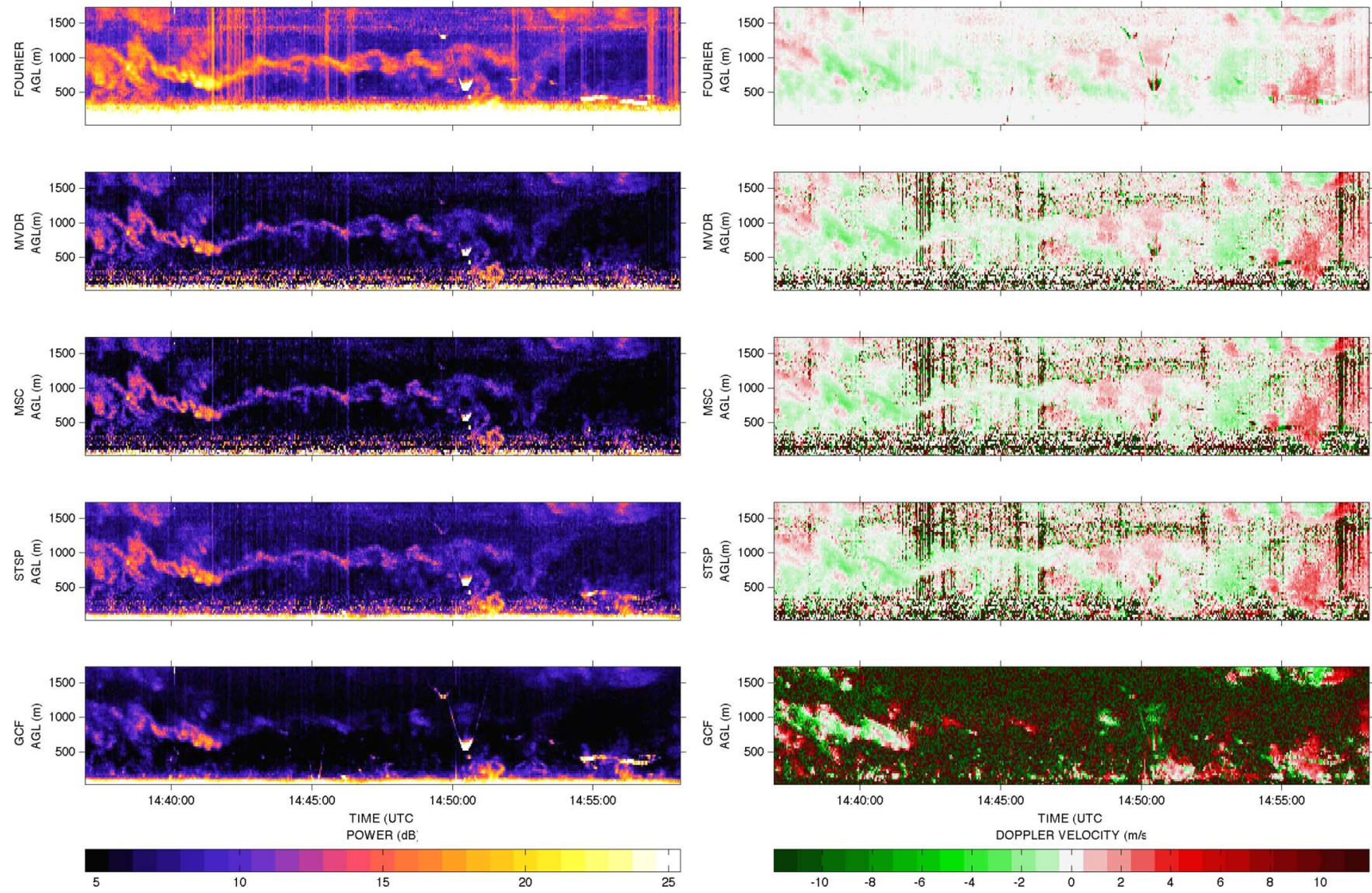

Fig. 10. 14:35-14:57 UTC on June 15, 2003. The power and Doppler velocity fields plotted are obtained using Fourier, GCF, MVDR, MSC, and STSP. The controlled parameters used in processing the data are NPTS $=4$ and $\epsilon=0.01$. The GCF is emulated by subtracting the mean value.

of echoes from ground targets are located below $500 \mathrm{~m}$ and above $1250 \mathrm{~m}$ which are present over the entire measurement time. These echoes are characterized by strong power values and near-zero Doppler velocity. In these images, the observed difference in power of the elevated and near-surface echoes is linked to the proximity difference to the receivers between the two ground targets. Examples of intermittent interference are the vertical power flashes that are characterized by powerful pulses of electromagnetic energy that span all the gates and also have near-zero Doppler velocity values. Sources of the intermittent interference are possibly the nearby cellular transmission towers, which operate at a frequency near $915 \mathrm{MHz}$. 
The examples of weather and nonweather echoes demonstrate the variety of scattering and interference sources that exist in the environmental field even for boundary layer profiling radars.

Since NPTS $=4$, a large diagonal load constraint of $\epsilon=$ 0.01 was applied to obtain results with low expected bias values for MVDR and MSC. As a consequence, the difference obtained between the three SLC techniques is small, and there is not much difference between the results obtained. A significant difference was observed between the SLC and the GCF techniques. The weather features are spatially continuous and have higher frequencies of low Doppler velocities with the SLC techniques, whereas they are intermittent and consist of larger Doppler velocities with the GCF technique. Examples of these differences are the power losses observed in the weather features between 500 and $1000 \mathrm{~m}$ where weather features appear like bubbles for the GCF technique as compared with a continuous flow for the SLC techniques.

\section{CONCLUSION}

The performance in terms of bias and standard deviation of the retrieved power for the MVDR, MSC, and STSP techniques was examined via simulation for the variations of $\epsilon, \mathrm{CSR}$, SNR, NPTS, and $\sigma_{\mathrm{c}}$. A comparison was made between a contaminated field with $\epsilon=0.1, \mathrm{CSR}=30 \mathrm{~dB}, \mathrm{SNR}=70 \mathrm{~dB}$, $\sigma_{\mathrm{c}}=0.1 \mathrm{~m} \cdot \mathrm{s}^{-1}$, and NPTS $=8$, and comparison was made to the same field without clutter contamination obtained using Fourier beamforming. The results obtained for the variation of $\epsilon$ showed that a balance between elevation angle and diagonal loading was required to optimally retrieve the weather signal. A degradation of performance was observed with increasing CSR for the MVDR and MSC techniques. The performance for STSP improved when CSR increased from 0 to $10 \mathrm{~dB}$, stayed approximately constant for CSR between 10 and $30 \mathrm{~dB}$, and degraded when CSR increased from 30 to $50 \mathrm{~dB}$. The performance of the three SLC techniques was approximately constant for SNR levels above $10 \mathrm{~dB}$ for the simulated parameters, and a degraded performance was observed only when the SNR was at this level. At low NPTS, a large diagonal loading value was required to retrieve the weather signal for MVDR and MSC. Diagonal loading values below the optimal value produced results for these techniques with negative bias power, whereas diagonal loading values above the optimal value produced results with residual clutter. At large NPTS, a lower diagonal loading was required, but the results using STSP were worse compared with that obtained at lower NPTS. Variation in $\sigma_{\mathrm{c}}$ produced a spread in the correlation matrix and resulted in a similar effect as seen in the results obtained using large NPTS.

The SLC techniques were also applied to the real data of boundary layer scattering with the TEP obtained between 14:35 and 14:57 UTC on June 15, 2003, using the constraints of NPTS $=4$ and $\epsilon=0.01$. The field was also obtained using a mean filter that acted as a GCF. Since a small $\epsilon$ was used, the difference between the retrieved results using the SLC techniques is small. However, a significant difference was observed in the retrieved fields between the SLC and GCF techniques. The weather features are spatially continuous and have higher frequencies of low Doppler velocities with the SLC techniques, whereas they are intermittent and consist of larger Doppler velocities with the GCF technique.

Given the increasing computational capacity of modern computers, implementation of more demanding algorithms will become a real possibility over the coming years. As a result, future clutter mitigation research can include studies of algorithms which exploit fully adaptive array designs. As has been shown in this paper, SLC solutions reduce the computational burden by adapting only a limited number of low-gain subarrays. A fully adaptive array has the potential of more aggressive spatial filtering of challenging nonstationary clutter targets that are near or in the main lobe of the antenna. As an example, a project is currently underway at The University of Oklahoma to explore the advantages of spatial filtering using fully adaptive arrays for the mitigation of wind turbines. With the nation's goal of generating $20 \%$ of its energy needs from renewable resources by the year 2020, clutter contamination by large wind turbines will become an increasingly important issue, which may be addressed by adaptive spatial filtering methods using phased arrays.

\section{ACKNOWLEDGMENT}

The authors would like to thank S. Frasier, F. Lopez-Dekker, and M. Hoffman for their contribution to the original TEP data.

\section{REFERENCES}

[1] D. Forsyth, J. Kimpel, D. Zrnic, S. Sandgathe, R. Ferek, J. Heimmer, T. M. J. Crain, J. Belville, and W. Benner, "Update on the National Weather Radar Testbed (phased-array)," in Proc. 23rd Conf. IIPS, San Antonio, TX, Jan. 14-18, 2007.

[2] T.-Y. Yu, M. Orescanin, C. Curtis, D. Zrnic, and D. Forsyth, "Beam multiplexing using the phased-array weather radar," J. Atmos. Ocean. Technol., vol. 24, no. 4, pp. 616-626, Apr. 2007.

[3] M. Yeary, B. McGuire, Y. Zhai, D. Forsyth, W. Benner, and G. Torok, "Target tracking at the National Weather Radar Testbed: A progress report on detecting and tracking aircraft," in Proc. 23rd Conf. IIPS, San Antonio, TX, Jan. 14-18, 2007.

[4] G. Zhang, R. Doviak, and X. Chen, "Spaced-antenna interferometry to detect discrete objects and sub-volume inhomogeneities of reflectivity," in Proc. 33rd Conf. Radar Meteorology, Cairns, Australia, Aug. 6-10, 2007.

[5] G. Zhang and R. Doviak, "Spaced-antenna interferometry to measure crossbeam wind, shear, and turbulence: Theory and formulation," J. Atmos. Ocean. Technol., vol. 24, no. 5, pp. 791-805, May 2007.

[6] M. Teshiba, T. Yu, G. Crain, and M. Xue, "A monopulse system: Applications for weather radar observations," in Proc. 33rd Conf. Radar Meteorology, Cairns, Australia, Aug. 6-10, 2007.

[7] B. L. Cheong, R. D. Palmer, C. D. Curtis, T.-Y. Yu, D. S. Zrnic, and D. Forsyth, "Refractivity retrieval using the phased-array radar: First results and potential for multimission operation," IEEE Trans. Geosci. Remote Sens., vol. 46, no. 9, pp. 2527-2537, Sep. 2008.

[8] P. Heinselman, D. Priegnitz, K. Manross, T. Smith, and R. Adams, "Rapid sampling of severe storms by the National Weather Radar Testbed Phased Array Radar," Weather Forecast., 2008. to be published.

[9] T. Smith, P. Heinselman, and D. Priegnitz, "Characteristics of microburst events observed with the National Weather Radar Testbed Phased Array Radar," in Proc. 23rd Conf. IIPS, San Antonio, TX, Jan. 14-18, 2007.

[10] B. Cheong, M. Hoffman, R. Palmer, S. Frasier, and F. Lopez-Dekker, "Phased-array design for biological clutter rejection: Simulation and experimental validation," J. Atmos. Ocean. Technol., vol. 23, no. 4, pp. 585598, Apr. 2006.

[11] R. Palmer, B. Cheong, M. Hoffman, S. Frasier, and F. Lopez-Dekker, "Observations of the small-scale variability of precipitation using an imaging radar," J. Atmos. Ocean. Technol., vol. 22, no. 8, pp. 1122-1137, Aug. 2005.

[12] H. Luce, M. Crochet, and F. Dalaudier, "Temperature sheets and aspect sensitive radar echoes," Ann. Geophys., vol. 19, no. 8, pp. 889-920, Aug. 2001. 
[13] J. Röttger and M. Larsen, UHF/VHF Radar Techniques for Atmospheric Research and Wind Profiler Applications, ser. Radar in Meteorology: Battan Memorial and 40th Anniversary. Boston, MA: AMS, 1980, ch. 21a, pp. 235-281.

[14] E. Kudeki and R. Woodman, "A poststatistics steering technique for MST radar applications," Radio Sci., vol. 25, no. 4, pp. 591-594, Aug. 1990.

[15] D. Hysell, "Radar imaging of equatorial F region irregularities with maximum entropy interferometry," Radio Sci., vol. 31, no. 6, pp. 1567-1578, 1996.

[16] J. Capon, "High-resolution frequency-wavenumber spectrum analysis," Proc. IEEE, vol. 57, no. 8, pp. 1408-1418, Aug. 1969.

[17] R. Palmer, S. Gopalam, T.-Y. Yu, and S. Fukao, "Coherent radar imaging using Capon's method," Radio Sci., vol. 33, no. 6, pp. 1585-1598, 1998.

[18] R. Palmer, M. Larsen, E. Sheppard, S. Fukao, M. Yamamoto, T. Tsuda, and S. Kato, "Poststatistic steering wind estimation in the troposphere and lower stratosphere," Radio Sci., vol. 28, no. 3, pp. 261-272, 1993.

[19] T.-Y. Yu, R. Palmer, and P. Chilson, "An investigation of scattering mechanisms and dynamics in PMSE using coherent radar imaging," J. Atmos. Sol.-Terr. Phys., vol. 63, no. 17, pp. 1797-1810, Nov. 2001

[20] I. Reed, J. Goldstein, X. Yu, and P. Singer, "Multidisciplinary perspective on adaptive sensor array processing," Proc. Inst. Electr. Eng.-Radar Sonar Navigation, vol. 146, no. 5, pp. 221-234, Oct. 1999.

[21] B. Van Veen and K. Buckley, "Beamforming: A versatile approach to spatial filtering," IEEE ASSP Mag., vol. 5, no. 2, pp. 4-24, Apr. 1988.

[22] H. Krim and M. Viberg, "Two decades of array signal processing research-The parametric approach," IEEE Signal Process. Mag., vol. 13, no. 4, pp. 67-94, Jul. 1996.

[23] I. PopStefanija, J. Knorr, P. Buczynski, and B. Bluth, "Advanced weather surveillance algorithms and techniques using a rapid scanning X-band radar-First results," in Proc. 32nd Conf. Radar Meteorology, Albuquerque, NM, Oct. 22-29, 2005.

[24] S. Torres and D. Zrnic, "Ground clutter canceling with a regression filter," J. Atmos. Ocean. Technol., vol. 16, no. 10, pp. 1364-1372, Oct. 1999.

[25] J. Jordan, R. Lataitis, and D. Carter, "Removing ground and intermittent clutter contamination from wind profiler signals using wavelet transforms," J. Atmos. Ocean. Technol., vol. 14, no. 6, pp. 1280-1297, Dec. 1997.

[26] A. Siggia and R. Passarelli, Jr., "Gaussian model adaptive processing (GMAP) for improved ground clutter cancellation and moment calculation," SIGMET, Inc., Westford, MA, MS-NR: ERAD3-P-00117, 2005.

[27] R. Passarelli, Jr., "Parametric estimation of Doppler spectral moments: An alternative ground clutter rejection technique," J. Atmos. Ocean. Technol., vol. 22, no. 5, pp. 850-857, May 1983.

[28] A. Rhyzhkov and D. Zrnic, "Polarimetric rainfall estimation in the presence of anomalous propagation," J. Atmos. Ocean. Technol., vol. 15, no. 6, pp. 1320-1330, Dec. 1998.

[29] R. Doviak and D. Zrnic, "Siting of Doppler weather radars to shield ground targets," IEEE Trans. Antennas Propag., vol. AP-33, no. 7, pp. 685-689, Jul. 1985.

[30] R. Nitzberg, "Clutter Maps CFAR analysis," IEEE Trans. Aerosp. Electron. Syst., vol. AES-22, no. 4, pp. 419-421, Jul. 1986.

[31] H. Rohling, "Radar CFAR thresholding in clutter and multiple target situations," IEEE Trans. Aerosp. Electron. Syst., vol. AES-19, no. 4, pp. 608621, Jul. 1983

[32] B. Isom, R. Palmer, G. Secrest, R. Rhoton, D. Saxion, J. Winslow, J. Reed, T. Crum, and R. Vogt, "Characterization and mitigation of wind turbine clutter on weather radars," J. Atmos. Ocean. Technol., 2008. submitted for publication.

[33] P. Stoica and R. Moses, Spectral Analysis of Signals. Upper Saddle River, NJ: Prentice-Hall, 2005.

[34] D. Johnson, Array Signal Processing: Concepts and Techniques. Englewood Cliffs, NJ: Prentice-Hall, 2003.

[35] H. Van Trees, Optimum Array Processing: Part IV. New York: Wiley, 2002.

[36] D. Manolakis, V. Ingle, and S. Kogon, Statistical and Adaptive Signal Processing: Spectral Estimation, Signal Modeling, Adaptive Filtering and Array Processing. Boston, MA: McGraw-Hill, 2000.

[37] R. Monzingo, T. Miller, and W. Thomas, Introduction to Adaptive Arrays. Raleigh, NC: SciTech Publishing, 2004

[38] N. Fourikis, Advanced Array Systems, Applications and RF Technologies. San Diego, CA: Academic, 2000.

[39] P. Howells, "Intermediate frequency side-lobe canceller," U.S. Patent 3202990 , Aug. 24, 1965.

[40] B. Farhang-Boroujeny, Adaptive Filters: Theory and Application. New York: Wiley, 1998.

[41] S. Haykin, Adaptive Filter Theory, 3rd ed. Englewood Cliffs, NJ: Prentice-Hall, 1996.
[42] B. Carlson, "Covariance matrix estimation errors and diagonal loading in adaptive arrays," IEEE Trans. Aerosp. Electron. Syst., vol. 24, no. 4, pp. 397-401, Jul. 1988.

[43] B. Widrow, P. Mantey, L. Griffiths, and B. Goode, "Adaptive antenna systems," Proc. IEEE, vol. 55, no. 12, pp. 2143-2159, Dec. 1967.

[44] S. Ellingson and G. Hampson, "A subspace-tracking approach to interference nulling for phased array-based radio telescopes," IEEE Trans. Antennas Propag., vol. 50, no. 1, pp. 25-30, Jan. 2002.

[45] B. Cheong, R. Palmer, and M. Xue, "A time-series weather radar simulator based on high-resolution atmospheric models," J. Atmos. Ocean. Technol., vol. 25, no. 2, pp. 230-243, Feb. 2008.

[46] M. Xue, K. Droegemeier, and V. Wong, "The Advanced Regional Prediction System (ARPS) - A multi-scale nonhydrostatic atmospheric simulation and prediction model. Part I: Model dynamics and verification," Meteor. Atmos. Phys., vol. 75, no. 3/4, pp. 161-193, 2000.

[47] M. Xue, K. Droegemeier, V. W. A. Shapiro, K. Brewster, F. Carr, D. Weber, Y. Liu, and D. Wang, "The Advanced Regional Prediction System (ARPS) - A multi-scale nonhydrostatic atmospheric simulation and prediction model. Part II: Model physics and applications," Meteor. Atmos. Phys., vol. 76, no. 3/4, pp. 143-165, 2001

[48] P. Ray, B. Johnson, K. Johnson, J. Bradberry, J. Stephens, K. Wagner, R. Wilhelmson, and J. Klemp, "The morphology of several tornadic storms on 20 May 1977," J. Atmos. Sci., vol. 38, no. 8, pp. 1643-1663, Aug. 1981.

[49] J. Mead, G. Hopcraft, S. Frasier, B. Pollard, C. Cherry, D. Schaubert, and R. McIntosh, "A volume-imaging radar wind profiler for atmospheric boundary layer turbulence studies," J. Atmos. Ocean. Technol., vol. 15, no. 4, pp. 849-859, Aug. 1998.

[50] J. Doran, J. Fast, and J. Horel, "The VTMX 2000 Campaign," Bull. Amer. Meteorol. Soc., vol. 83, no. 4, pp. 537-551, Apr. 2002.

[51] B. Pollard, S. Khanna, S. Frasier, J. Wyngaard, D. Thomson, and R. McIntosh, "Local structure of the convective boundary layer from a volume-imaging radar," J. Atmos. Sci., vol. 57, no. 14, pp. 2281-2296, Jul. 2000.

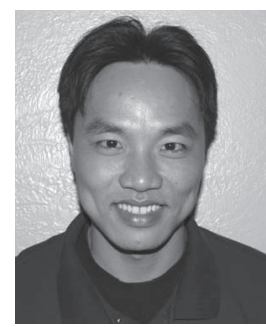

Khoi D. Le received the B.S. and M.S. degrees in electrical engineering from the University of Nebraska, Lincoln, in 1999 and 2001, respectively. $\mathrm{He}$ is currently working toward the Ph.D. degree in electrical engineering in the School of Electrical and Computer Engineering, The University of Oklahoma, Norman.

His research interests include array processing and digital signal processing applied to weather radar applications.

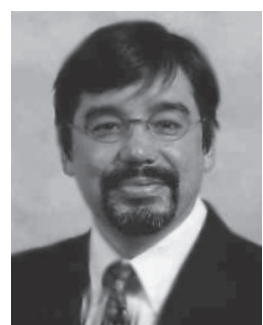

Robert D. Palmer (S'86-M'86-SM'93) received the $\mathrm{Ph} . \mathrm{D}$. degree in electrical engineering from The University of Oklahoma (OU), Norman, in 1989.

From 1989 to 1991, he was a Postdoctoral Fellow with the RadioAtmospheric Science Center, Kyoto University, Kyoto, Japan, where his major accomplishments were the development of novel interferometric radar techniques for studies of the lower and middle atmospheres. From 1993 to 2004, he was a Member of the Faculty with the Department of Electrical Engineering, University of Nebraska, Lincoln, where his interests broadened into areas including wireless communications, remote sensing, and pedagogy. He was a Research Associate with the Physics and Astronomy Department, Clemson University, Clemson, SC, where he continued his work with atmospheric radar. He is currently the Tommy C. Craighead Chair with the School of Meteorology, OU, where he is also an Adjunct Professor with the School of Electrical and Computer Engineering. He is the Director of OU's interdisciplinary Atmospheric Radar Research Center, which is the focal point for the weather-radar research and educational activities on the Norman campus. Since he joined OU, his research interests have been focused primarily on the application of advanced radarsignal-processing techniques to observations of severe weather, particularly related to phased-array radars and other innovative system designs. He has published widely in the area of radar remote sensing of the atmosphere, with an emphasis on generalized imaging problems, spatial filter design, and clutter mitigation using advanced array/signal-processing techniques. 


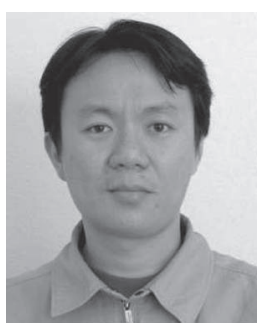

Boon Leng Cheong received the B.S., M.S., and $\mathrm{Ph} . \mathrm{D}$. degrees in electrical engineering from the University of Nebraska, Lincoln, in 1999, 2002, and 2005 , respectively.

Since 2005, he has been a Postdoctoral Fellow with the School of Meteorology, The University of Oklahoma, Norman. Besides the work on refractivity retrieval, his research interests include numerical radar simulations, array processing using multiple receivers, and real-time signal processing applied to radar and acoustic applications. Recently, he has developed a radar time-series simulator that utilizes realistic atmospheric fields.

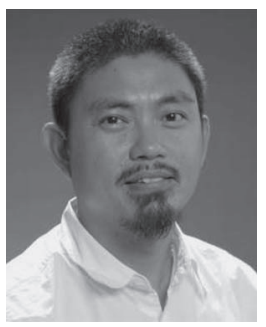

Tian-You Yu received the B.S. degree in atmospheric physics and the M.S. degree in space science from the National Central University, Taoyuan, Taiwan, R.O.C., and the Ph.D. degree in electrical engineering from the University of Nebraska, Lincoln, in 2000

He was a Postdoctoral Fellow with the National Center for Atmospheric Research. He had worked on various types of proler radars to study atmospheric dynamics from the boundary layer to the mesosphere. He is currently an Assistant Professor with the School of Electrical and Computer Engineering, The University of Oklahoma, Norman. He has a wide range of interests that include digital signal processing and atmospheric applications through radar observations. His current research interests include the development of novel and sophisticated radar technologies to improve radar measurement and the advancement of the fundamental knowledge of meteorological phenomena.

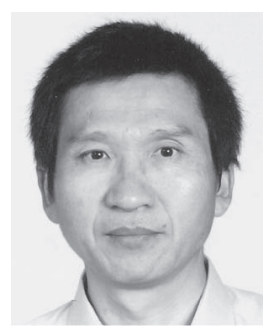

Guifu Zhang (S'97-M'98-SM'02) received the B.S. degree in physics from Anhui University, Hefei, China, in 1982, the M.S. degree in radio physics from Wuhan University, Wuhan, China, in 1985, and the Ph.D. degree in electrical engineering from the University of Washington, Seattle, in 1988.

From 1985 to 1993, he was an Assistant and Associate Professor with the Space Physics Department, Wuhan University. In 1989, he was a Visiting Scholar with the Communication Research Laboratory, Japan. From 1993 to 1998, he studied and worked with the Department of Electrical Engineering, University of Washington, where he was first a Visiting Scientist and later a Ph.D. student. He was a Scientist with the National Center for Atmospheric Research. He is currently with the School of Meteorology, The University of Oklahoma, Norman. His research interests include the modeling of wave propagation and scattering in geophysical media, and the development of remote-sensing techniques for environmental monitoring. He is currently interested in radar polarimetry and interferometry for weather quantification and forecasting.

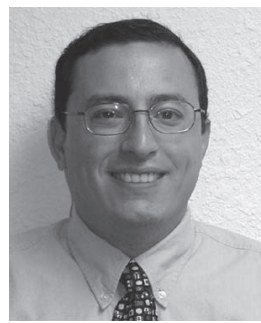

Sebastian M. Torres (S'99-M'01) received the B.S. degree in electrical engineering from the $\mathrm{Na}-$ tional University of Mar del Plata, Mar del Plata, Argentina, in 1995, and the M.S. and Ph.D. degrees in electrical engineering from The University of Oklahoma, Norman, in 1997 and 2001, respectively.

From 1991 to 1996, he was a Research Associate with the Signal Processing and Measurements Laboratory, National University of Mar del Plata. Since 1997, he has been with the Cooperative Institute for Mesoscale Meteorological Studies, The University of Oklahoma. He is also currently a Research Scientist with the NOAA/OAR National Severe Storms Laboratory, Norman. His research interests include applications of signal processing to radar meteorology and their implementation in real-time embedded systems.

Dr. Torres has received the Department of Commence Silver Medal as a member of the Stormscale Research and Applications Division at the National Severe Storms Laboratory for making significant enhancements to the NEXRAD system and the 2003-2004 Office of Oceanic and Atmospheric Research Outstanding Scientific Paper Award. 